\title{
Mesoscopic superposition of states with sub-Planck structures in phase space
}

\author{
G. S. Agarwal and P. K. Pathak \\ Physical Research Laboratory, Navrangpura, Ahmedabad-380 009, India \\ (Received 10 December 2003; revised manuscript received 18 May 2004; published 11 November 2004)
}

\begin{abstract}
We propose the cavity quantum electrodynamics method, using dispersive interaction between atoms and a high-quality cavity to realize the mesoscopic superposition of coherent states that would exhibit sub-Planck structures in phase space, i.e., the structures at a scale smaller than the Plank's constant $(\hbar)$. These structures are direct signatures of quantum coherence and are formed as a result of interference between the two superposed cat states. In particular we focus on a superposition involving four coherent states. We show interferences in the conditional measurements involving two atoms.
\end{abstract}

DOI: 10.1103/PhysRevA.70.053813

PACS number(s): 42.50.Gy, 32.80.Qk

\section{INTRODUCTION}

In recent times mesoscopic superposition of states has attracted a great deal of attention as these superpositions exhibit very important interference effects [1-3] many of which have now been realized experimentally [4-8]. The simplest superposition would consist of two coherent states one centered at $\alpha$ and the other at $-\alpha$. Such a state is known to be an eigenstate of the operator $a^{2}$. It has been known that the passage of a single mode of the field in a coherent state through a Kerr medium could produce such a state [9-11]. In an earlier work [9], it was shown that a variety of other superpositions can be produced by a Kerr medium. In particular, one can produce eigenstates of the operator $a^{4}$. Such eigenstates are superpositions of four coherent states. However, an efficient production of such states would require a large Kerr nonlinearity which is not available though some proposals for the enhancement of the Kerr nonlinearity exist [12]. The existence of such superpositions is closely connected to the occurrence of fractional revivals in the nonlinear dynamics of quantum systems [8,13-15]. In particular, a fractional revival of order $1 / 4$ can produce a superposition of four coherent states. However, instead of pursuing the production using Kerr medium, we propose to use cavity QED methods. We note that Haroche and co-workers [3,5,6] showed how cavity quantum electrodynamics can be used to produce a superposition of two mesoscopic states. It turns out that one can have a fairly large dispersive interaction in high-quality cavities. This high dispersion has been utilized by several authors $[16,17]$ to produce a variety of entangled states and nonclassical superpositions including a superposition of four coherent states. In this paper we show how to prepare superpositions of four coherent states by using dispersive interaction in a high-quality cavity. This study is motivated by a recent finding of Zurek [18] that a proper superposition of four coherent states which he refers to as a compass state, can exhibit regions in phase space with subPlanck structures, i.e., the area of the variations of the two quadratures can be much smaller than $\hbar$. We demonstrate how the results of conditional measurements on three atoms passing in succession, through a high- $Q$ cavity, can yield information on such a compass state.

\section{COMPASS STATE FOR THE RADIATION FIELD}

Consider a single-mode radiation field specified by the annihilation and creation operators $a$ and $a^{\dagger}$. Let $|\alpha\rangle$ be a coherent state for the field with amplitude $\alpha$. The most commonly studied superpositions are of the form

$$
|\psi\rangle \sim|\alpha\rangle+\left|e^{i \theta} \alpha\right\rangle .
$$

Here $\theta$ is an arbitrary phase. Extensive literature on this state exists. It is well known $[1,2]$ that the quantum character of this state is reflected in the regions of phase space where the Wigner function becomes negative. The area of the negative region is of the order of Planck constant. There are several methods of producing such a state [3,7-9]. Zurek [18] has studied a superposition state of four Gaussian wave packets

$$
\psi(x) \sim \exp \left\{-\frac{\left(x-x_{0}\right)^{2}}{\xi^{2}}+\frac{i p_{0} x}{\hbar}\right\},
$$

with one each placed in the east, west, north, and south direction in the phase space and calculated the Wigner function for such a state, defined by

$$
W(x, p)=\frac{1}{2 \pi \hbar} \int e^{i p y / \hbar} \psi\left(x-\frac{y}{2}\right) \psi *\left(x+\frac{y}{2}\right) d y .
$$

He found that it exhibits negative regions in phase space as well as structures with areas which could be much smaller than Planck's constant. Since coherent states correspond to Gaussian wave packets, in the following we consider a superposition of four coherent states of the form

$$
|\phi\rangle=N(|\alpha\rangle+|i \alpha\rangle+|-\alpha\rangle+|-i \alpha\rangle),
$$

where $N$ is the normalization constant and $\alpha$ is complex. The Wigner function for any state $|\phi\rangle$ can be obtained using coherent states as [19]

$$
W\left(\gamma, \gamma^{*}\right)=\frac{2}{\pi^{2}} e^{2|\gamma|^{2}} \int\langle-\beta \mid \phi\rangle\langle\phi \mid \beta\rangle e^{-2\left(\beta \gamma^{*}-\beta^{*} \gamma\right)} d^{2} \beta .
$$

For the state (4) the Wigner function is found to be 


$$
\begin{aligned}
W\left(\gamma, \gamma^{*}\right)= & |N|^{2} \frac{4 e^{-2|\gamma|^{2}}}{\pi}\left[2 e^{-2|\alpha|^{2}} \cosh \left\{(1+i) \alpha \gamma^{*}+(1-i) \alpha^{*} \gamma\right\} \cosh \left\{(1-i) \alpha \gamma^{*}+(1+i) \alpha^{*} \gamma\right\}+2 \cos \left\{(1+i) \alpha \gamma^{*}\right.\right. \\
& \left.+(1-i) \alpha^{*} \gamma\right\} \cos \left\{(1-i) \alpha \gamma^{*}+(1+i) \alpha^{*} \gamma\right\}+e^{-\left[|\alpha|^{2}-(1+i) \alpha \gamma^{*}-(1-i) \alpha^{*} \gamma\right]} \cos \left\{|\alpha|^{2}-(1+i) \alpha \gamma^{*}-(1-i) \alpha^{*} \gamma\right\} \\
& +e^{-\left[|\alpha|^{2}-(1-i) \alpha \gamma^{*}-(1+i) \alpha^{*} \gamma\right]} \cos \left\{|\alpha|^{2}-(1-i) \alpha \gamma^{*}-(1+i) \alpha^{*} \gamma\right\}+e^{-\left[|\alpha|^{2}+(1+i) \alpha \gamma^{*}+(1-i) \alpha^{*} \gamma\right]} \cos \left\{|\alpha|^{2}+(1+i) \alpha \gamma^{*}\right. \\
& \left.+(1-i) \alpha^{*} \gamma\right\}+e^{\left.-\left[|\alpha|^{2}+(1-i) \alpha \gamma^{*}+(1+i) \alpha^{*} \gamma\right)\right] \cos \left\{|\alpha|^{2}+(1-i) \alpha \gamma^{*}+(1+i) \alpha^{*} \gamma\right\} .}
\end{aligned}
$$

Each cosine term in Eq. (6) arises from the interference of a pair of coherent states in the superposition state (4). The sub-Plank structures arise from further interference of two cosine terms which come from the diagonal pairs. The first two terms in Eq. (6) are such terms coming from the diagonal pairs $|\alpha\rangle,|-\alpha\rangle$ and $|i \alpha\rangle,|-i \alpha\rangle$. The first term is significant for smaller values of $|\alpha|$ and shows exponential decrease in the Wigner function away from the center and the second term which is significant for larger values of $|\alpha|$ shows the interference pattern in the central region $(\gamma \rightarrow 0)$. In Figs. 1 and 2, we plot the Wigner function for some typical values of $|\alpha|$. We found that for smaller values of $|\alpha|$ (Fig. 1), the central part has a continuum and no other structures appear but for larger values of $|\alpha|$ (Fig. 2), a chess board pattern as noticed earlier by Zurek appears in the central region. The reason for the disappearance of the interference pattern in the central region for smaller values of $|\alpha|$ is because in this case the coherent states overlap to a large extent so the interference effects are not visible.

A natural question is how to produce the state (4). In what follows we show how the methods of cavity quantum electrodynamics $[3,5]$ can be generalized to produce the state (4).

\section{GENERATION OF THE COMPASS STATE USING DISPERSIVE INTERACTION BETWEEN ATOMS AND CAVITY}

Consider a single-mode high- $Q$ cavity containing a small amount of a coherent field so that the initial state of the

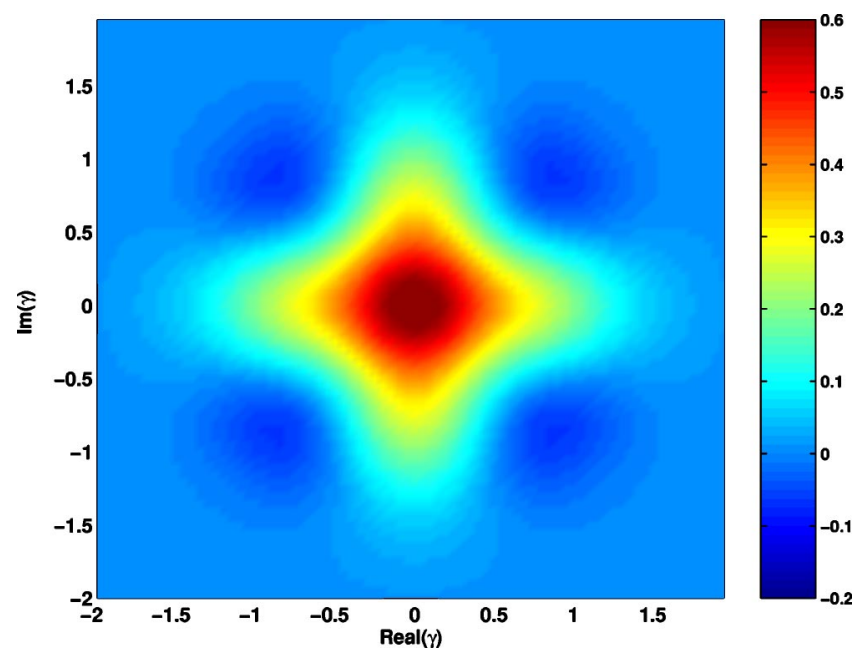

FIG. 1. (Color online) The Wigner function for mesoscopic superposition state $N(|\alpha\rangle+|-\alpha\rangle+|i \alpha\rangle+|-i \alpha\rangle)$ for $|\alpha|=1$. cavity field is $|\alpha\rangle$. Let $\omega_{c}$ be the cavity frequency. Consider the passage of a two level atom with the excited and ground states $|e\rangle$ and $|g\rangle$ with transition frequency $\omega$. The atom is initially prepared in a superposition state

$$
|\Phi\rangle=c_{e}|e\rangle+c_{g}|g\rangle .
$$

In a frame rotating with the atomic transition frequency $\omega$, the interaction Hamiltonian is given by

$$
H=\hbar \delta a^{\dagger} a+\hbar g\left(|e\rangle\langle g|a+| g\rangle\langle e| a^{\dagger}\right), \delta=\left(\omega_{c}-\omega\right) .
$$

We assume that we are working in the dispersive limit so that $\delta$ is large. We can then do a second order perturbation theory and obtain an effective Hamiltonian

$$
H \simeq \hbar \delta a^{\dagger} a+\phi_{0} \hbar a^{\dagger} a|g\rangle\left\langle g\left|-\phi_{0} \hbar a a^{\dagger}\right| e\right\rangle\langle e|,
$$

where the parameter $\phi_{0}$ is equal to $g^{2} / \delta$. Physically it gives the shift of the excited state in the absence of any cavity field. Under the effect of the Hamiltonian (9), the states evolve as

$$
\begin{gathered}
|g, n\rangle \rightarrow e^{-i n \phi_{0} \tau-i n \delta \tau}|g, n\rangle, \\
|e, n\rangle \rightarrow e^{i(n+1) \phi_{0} \tau-i n \delta \tau}|e, n\rangle,
\end{gathered}
$$

where $\tau$ is the interaction time. Using Eq. (10), we easily obtain the evolution of a field in a coherent state $|\alpha\rangle$

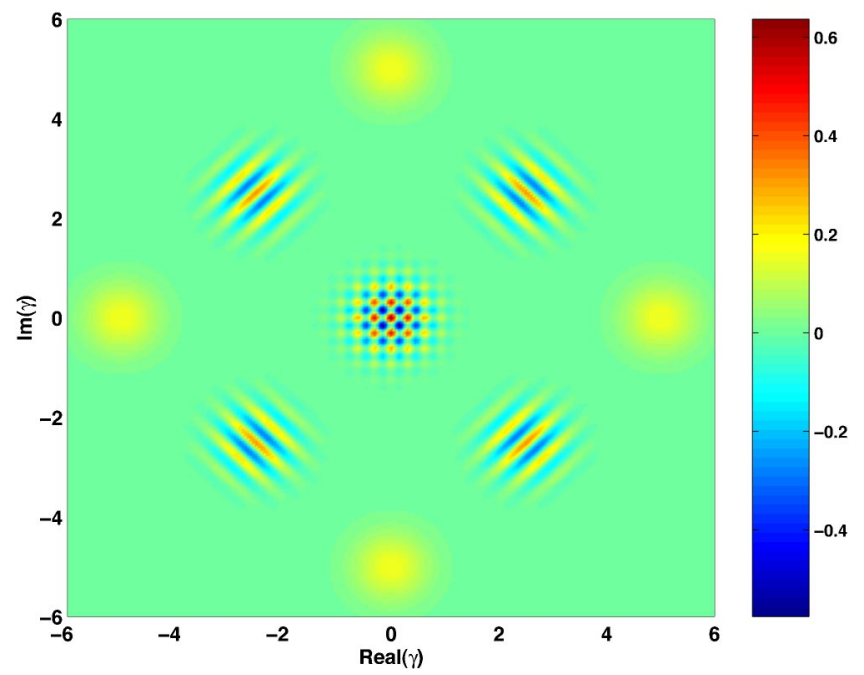

FIG. 2. (Color online) The Wigner function for mesoscopic superposition state $N(|\alpha\rangle+|-\alpha\rangle+|i \alpha\rangle+|-i \alpha\rangle)$ for $|\alpha|=5$. 


$$
\begin{gathered}
|g, \alpha\rangle \rightarrow\left|g, \alpha e^{-i \phi-i \delta \tau}\right\rangle, \\
|e, \alpha\rangle \rightarrow e^{i \phi}\left|e, \alpha e^{i \phi-i \delta \tau}\right\rangle, \quad \phi=\phi_{0} \tau .
\end{gathered}
$$

Therefore the atom field system in the state $|\Phi, \alpha\rangle$ will evolve into

$$
|\Phi, \alpha\rangle \rightarrow c_{e} e^{i \phi}\left|e, \alpha e^{i \phi-i \delta \tau}\right\rangle+c_{g}\left|g, \alpha e^{-i \phi-i \delta \tau}\right\rangle .
$$

The probability of detection of the atom in the state $|\psi\rangle$ $=\psi_{e}|e\rangle+\psi_{g}|g\rangle$ will be

$$
\begin{gathered}
P_{\psi}=\| c_{e} \psi_{e}^{*} e^{i \phi}\left|\alpha e^{i \phi-i \delta \tau}\right\rangle+c_{g} \psi_{g}^{*}\left|\alpha e^{-i \phi-i \delta \tau}\right\rangle \|^{2} \\
=\left|c_{e} \psi_{e}^{*}\right|^{2}+\left|c_{g} \phi_{g}^{*}\right|^{2}+2 \operatorname{Re}\left(c_{g}^{*} \psi_{g} c_{e} \psi_{e}^{*} e^{i \phi}\left\langle\alpha e^{-i(\phi+\delta \tau)} \mid \alpha e^{i(\phi-\delta \tau)}\right\rangle\right) .
\end{gathered}
$$

The last term in Eq. (14) yields the interference fringes. For the special case of the initial state and the detection state having equal superposition of the ground and the excited states $\left|c_{g}^{*} \psi_{g} c_{e} \psi_{e}^{*}\right|=1 / 4$. The visibility depends on the scalar product of two coherent states that are shifted in phase by $2 \phi$. The phase shift is a measure of the cavity interaction. Haroche and co-workers have used the above for the production and detection of mesoscopic superposition of the field states. In the present case the generated mesoscopic superposition is the state in Eq. (13) under the \|\| sign.

We next demonstrate how the compass state can be produced by following similar ideas. Let us write the state (12) in the form

$$
|\Phi, \alpha\rangle=f_{e}|e\rangle\left|\alpha_{e}\right\rangle+f_{g}|g\rangle\left|\alpha_{g}\right\rangle .
$$

Let us consider the passage of two atoms labeled as $A$ and $B$ in succession through the cavity. After the passage of the atom $A$ we get the state (15). Clearly the net state of the system consisting of two atoms $A, B$ and the cavity field would have the structure

$$
\begin{aligned}
|\Psi\rangle= & f_{e} h_{e}\left|e_{A}, e_{B}\right\rangle\left|\alpha_{e e^{\prime}}\right\rangle+f_{e} h_{g}\left|e_{A}, g_{B}\right\rangle\left|\alpha_{e g^{\prime}}\right\rangle+f_{g} h_{e}\left|g_{A}, e_{B}\right\rangle\left|\alpha_{g e^{\prime}}\right\rangle \\
& +f_{g} h_{g}\left|g_{A}, g_{B}\right\rangle\left|\alpha_{g g^{\prime}}\right\rangle .
\end{aligned}
$$

The joint detection of the atoms in the state $|\chi\rangle$ $\equiv \chi_{e e^{\prime}}\left|e_{A}, e_{B}\right\rangle+\chi_{e g^{\prime}}\left|e_{A}, g_{B}\right\rangle+\chi_{g e^{\prime}}\left|g_{A}, e_{B}\right\rangle+\chi_{g g^{\prime}}\left|g_{A}, g_{B}\right\rangle \quad$ will project state (16) to (unnormalized state)

$$
\begin{aligned}
\langle\chi \mid \Psi\rangle \equiv & |C\rangle=f_{e} h_{e} \chi_{e e^{\prime}}^{*}\left|\alpha_{e e^{\prime}}\right\rangle+f_{e} h_{g} \chi_{e g^{\prime}}^{*}\left|\alpha_{e g^{\prime}}\right\rangle+f_{g} h_{e} \chi_{g e^{\prime}}^{*}\left|\alpha_{g e^{\prime}}\right\rangle \\
& +f_{g} h_{g} \chi_{g g^{\prime}}^{*}\left|\alpha_{g g^{\prime}}\right\rangle .
\end{aligned}
$$

Clearly such a conditional detection reduces the state of the cavity field to a state which in general would be a mesoscopic superposition of four coherent sates $\left|\alpha_{i j}\right\rangle$. The value of $\alpha_{i j}$ can be read from Eq. (12):

$$
\begin{gathered}
\alpha_{e e^{\prime}}=\alpha_{0} e^{i \phi+i \phi^{\prime}}, \quad \alpha_{e g^{\prime}}=\alpha_{0} e^{i \phi-i \phi^{\prime}}, \\
\alpha_{g e^{\prime}}=\alpha_{0} e^{-i \phi+i \phi^{\prime}}, \quad \alpha_{g g^{\prime}}=\alpha_{0} e^{-i \phi-i \phi^{\prime}} ; \\
\phi=\frac{g_{A}^{2} \tau_{A}}{\delta}, \quad \phi^{\prime}=\frac{g_{B}^{2} \tau_{B}}{\delta} ;
\end{gathered}
$$

$$
\alpha_{0}=\alpha e^{-i \delta \tau-i \delta \tau^{\prime}} .
$$

Clearly by varying $\phi$ and $\phi^{\prime}$ we can produce a variety of superpositions. Consider, for example, $\phi=\pi / 4$ and $\phi^{\prime}$ $=\pi / 2$, then

$$
\begin{aligned}
& \alpha_{e e^{\prime}}=\alpha_{0} e^{3 i \pi / 4}, \quad \alpha_{e g^{\prime}}=\alpha_{0} e^{-i \pi / 4}, \\
& \alpha_{g e^{\prime}}=\alpha_{0} e^{i \pi / 4}, \quad \alpha_{g g^{\prime}}=\alpha_{0} e^{-3 i \pi / 4},
\end{aligned}
$$

so the state (17) is a compass state. The expansion coefficients in Eq. (17) depend on the initial preparation of the atoms $A$ and $B$ and the detection of these atoms. This is usually done by using two Ramsey zones before and after the cavity. Let us for simplicity assume that

$$
\begin{gathered}
\left|\Phi_{j}\right\rangle=\frac{1}{\sqrt{2}}\left(e^{i \eta_{j}}|e\rangle+e^{i \theta_{j}}|g\rangle\right) ; j=A, B, \\
|\chi\rangle=\left|\Phi_{A}^{\prime}\right\rangle\left|\Phi_{B}^{\prime}\right\rangle,
\end{gathered}
$$

where $\left|\Phi_{j}^{\prime}\right\rangle$ is obtained from $\left|\Phi_{j}\right\rangle$ by using $\eta_{j} \rightarrow \eta_{j}^{\prime}$ and $\theta_{j}$ $\rightarrow \theta_{j}^{\prime}$. Substituting values of $\alpha_{i j}$ from Eq. (19) we rewrite Eq. (17) as

$$
\begin{aligned}
|C\rangle= & \frac{1}{4}\left(e^{i\left(\eta_{1}+\eta_{2}+3 \pi / 4\right)}|-\alpha\rangle+e^{i\left(\eta_{1}+\theta_{2}+\pi / 4\right)}|\alpha\rangle+e^{i\left(\theta_{1}+\eta_{2}+\pi / 2\right)}|i \alpha\rangle\right. \\
& \left.+e^{i\left(\theta_{1}+\theta_{2}\right)}|-i \alpha\rangle\right),
\end{aligned}
$$

$$
\eta_{1}=\eta_{A}-\eta_{A}^{\prime}, \quad \eta_{2}=\eta_{B}-\eta_{B}^{\prime}, \quad \theta_{1}=\theta_{A}-\theta_{A}^{\prime}, \quad \theta_{2}=\theta_{B}-\theta_{B}^{\prime},
$$

we have also set $\alpha_{0}=\alpha e^{i \pi / 4}$. For $\theta_{1}=\eta_{1}+\pi / 4$ and $\theta_{2}=\eta_{2}$ $+\pi / 2$ the state (21) becomes the compass state (4)

$$
|C\rangle=\frac{1}{4} e^{i\left(\eta_{1}+\eta_{2}+3 \pi / 4\right)}(|-\alpha\rangle+|\alpha\rangle+|i \alpha\rangle+|-i \alpha\rangle) .
$$

It is clear that the probability of joint measurements on the atoms $A$ and $B$ would be

$$
P=\operatorname{Tr}_{c}\langle\chi \mid \psi\rangle\langle\psi \mid \chi\rangle,
$$

where $\operatorname{Tr}_{c}$ stands for tracing over the cavity field. Using Eqs. (17) and (20), we find the result

$$
\begin{aligned}
P= & \frac{1}{4}+\frac{1}{8} \operatorname{Re}\left(e^{i\left(\theta_{2}-\eta_{2}-\pi / 2\right)}\langle-\alpha \mid \alpha\rangle+e^{i\left(\theta_{1}-\eta_{1}-\pi / 4\right)}\langle-\alpha \mid i \alpha\rangle\right. \\
& +e^{i\left(\theta_{1}+\theta_{2}-\eta_{1}-\eta_{2}-3 \pi / 4\right)}\langle-\alpha \mid-i \alpha\rangle+e^{i\left(\theta_{1}-\eta_{l}-\pi / 4\right)}\langle\alpha \mid-i \alpha\rangle \\
& \left.+e^{i\left(\theta_{1}+\eta_{2}-\eta_{1}-\theta_{2}+\pi / 4\right)}\langle\alpha \mid i \alpha\rangle+e^{i\left(\theta_{2}-\eta_{2}-\pi / 2\right)}\langle i \alpha \mid-i \alpha\rangle\right) \\
\equiv & \langle C \mid C\rangle .
\end{aligned}
$$

In Fig. 3 we show $P$ as a function of phases of initial atomic state and the detected atomic state for $|\alpha|=1$. These interferences become less prominent for larger values of $|\alpha|$. The exact nature of interferences depends on the choice of the phase factors $\eta_{j}$ and $\theta_{j}$.

In order to explore the characteristics of the state (22), we have to bring a third atom $C$ and examine the probability of its detection in a given state. This would be similar to what was done in the experiment of Brune et al. [5] to study the 


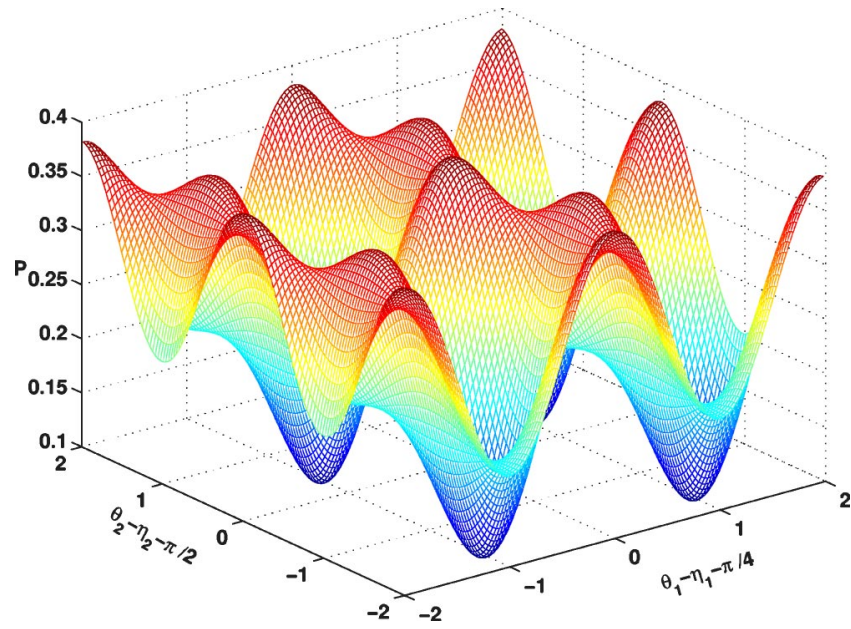

FIG. 3. (Color online) The probability $P$ for $|\alpha|^{2}=1$ is plotted with phases of the initial atomic state and the detected state. The scale along the $x$ axis and $y$ axis is in units of $\pi$.

cat state (the mesoscopic superposition of two coherent states). Another possibility would involve a probe atom interacting resonantly with the prepared field in the cavity as the compass state (4) involves photon number states which are multiples of four. We discuss in Appendix A the differences in the excitation probabilities for different states in the cavity.

Following the work of Davidovich et al. [3] we can examine the effect of detection efficiency on the preparation of the compass state. If one atom passes through the cavity undetected it will leave the cavity either in its excited state $|e\rangle$ or in its ground state $|g\rangle$, in both cases it will produce phase shift in all the superposed coherent states equally and as a result it cannot affect the compass state except rotating it in phase space. So in the experimental realization of such mesoscopic superposition high efficiency detection is not necessary. We relegate the details to Appendix B. We further note that more complex homodyne methods like the ones used in Ref. [4] can be employed to probe the phase space distributions associated with the compass state.

Next we consider effects of decoherence on the compass state (4). This can be done using the master equation

$$
\dot{\rho}=-\frac{\kappa}{2}\left(a^{\dagger} a \rho-2 a \rho a^{\dagger}+\rho a^{\dagger} a\right),
$$

where $\kappa$ is the cavity field decay parameter and we assume that the cavity is at zero temperature. For the initial state (4) we find the density matrix after time $t$ :

$$
\begin{aligned}
\rho(t)= & |N|^{2}\left[\left|\alpha_{t}\right\rangle\left\langle\alpha_{t}|+|-\alpha_{t}\right\rangle\left\langle-\alpha_{t}|+| i \alpha_{t}\right\rangle\left\langle i \alpha_{t}|+|-i \alpha_{t}\right\rangle\left\langle-i \alpha_{t}\right|+e^{-2|\alpha|^{2}\left(1-e^{-\kappa t}\right)}\left(\left|\alpha_{t}\right\rangle\left\langle-\alpha_{t}|+|-\alpha_{t}\right\rangle\left\langle\alpha_{t}|+| i \alpha_{t}\right\rangle\left\langle-i \alpha_{t}|+|-i \alpha_{t}\right\rangle\left\langle i \alpha_{t}\right|\right)\right. \\
& +e^{-|\alpha|^{2}(1-i)\left(1-e^{-\kappa t}\right)}\left(\left|\alpha_{t}\right\rangle\left\langle i \alpha_{t}|+|-i \alpha_{t}\right\rangle\left\langle\alpha_{t}|+|-\alpha_{t}\right\rangle\left\langle-i \alpha_{t}|+| i \alpha_{t}\right\rangle\left\langle-\alpha_{t}\right|\right)+e^{-|\alpha|^{2}(1+i)\left(1-e^{-\kappa t}\right)}\left(\left|i \alpha_{t}\right\rangle\left\langle\alpha_{t}|+| \alpha_{t}\right\rangle\left\langle-i \alpha_{t}\right|\right. \\
& \left.\left.+\left|-i \alpha_{t}\right\rangle\left\langle-\alpha_{t}|+|-\alpha_{t}\right\rangle\left\langle i \alpha_{t}\right|\right)\right] ; \alpha_{t}=\alpha e^{-\kappa t / 2}
\end{aligned}
$$

The coherence of the superposition decays as $e^{-2|\alpha|^{2}\left(1-e^{-\kappa t}\right)}$ which is $e^{-2|\alpha|^{2} \kappa t}$ in the limit $\kappa t \ll 1$. Thus the lifetime of the compass state will be $t_{c} / 2|\alpha|^{2}, t_{c}$ is the lifetime of the cavity field. So the lifetime for the compass state is the same as for a Schrodinger cat state (1).

\section{CONCLUSIONS}

We discussed the properties of the compass state for a radiation field as well as the methods for generating a compass state using the dispersive atom cavity interaction. We showed that the central interference pattern in the Wigner function for mesoscopic superposition of cat states appears for larger values of $|\alpha|$ and disappears for smaller values. The conditional measurements enable one to study some aspects of the mesoscopic superposition of coherent states. We have also discussed the effects of decoherence on the compass state as well as the effects of nonunity detection efficiency in the preparation of the compass state.

\section{APPENDIX A}

The compass state can be detected using the methods sensitive to its field statistics. For the compass-state photon dis- tribution is very special, it has a number states having a photon number in the integral multiple of four. The state (4) can be expressed in terms of the number of states as follows:

$$
|\phi\rangle=N \sum_{p} \frac{\alpha^{4 p}}{\sqrt{(4 p) !}} e^{-|\alpha|^{2} / 2}|4 p\rangle,
$$

where $p$ is an integer. We propose a simple method for detecting the compass state using a two level atom interacting resonantly with the cavity field as a probe. The Hamiltonian in the interaction picture is

$$
H=\hbar g\left(|e\rangle\left\langle g\left|a+a^{\dagger}\right| g\right\rangle\langle g|\right),
$$

where all symbols have their earlier defined meanings. Using the above interaction Hamiltonian we can calculate the probabilities of detection for the atom in its different states after passing through the cavity. The probabilities of detection if the atom enters the cavity in its lower state $|g\rangle$ and detected in its state $|g\rangle$ and $|e\rangle, P_{g}^{g}$ and $P_{g}^{e}$, respectively, are

$$
P_{g}^{g}=\sum_{p}\left|N \frac{\alpha^{4 p}}{\sqrt{(4 p) !}} e^{-|\alpha|^{2} / 2} \cos (2 g t \sqrt{p})\right|^{2},
$$



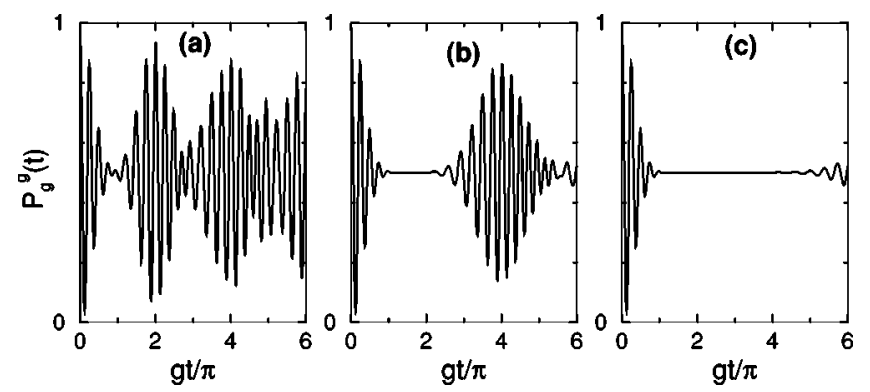

FIG. 4. The probability of detection of the atom in its ground state $|g\rangle$ for a cavity field in (a) compass state, (b) Schrodinger cat state $N_{0}(|\alpha\rangle+|-\alpha\rangle)$, and (c) coherent state $|\alpha\rangle$, for $|\alpha|^{2}=16$.

$$
P_{g}^{e}=\sum_{p}\left|N \frac{\alpha^{4 p}}{\sqrt{(4 p) !}} e^{-|\alpha|^{2} / 2} \sin (2 g t \sqrt{p})\right|^{2} .
$$

In Fig. 4 we show the comparison of detection probabilities for the cavity field in the compass state, Schrodinger cat state $N_{0}(|\alpha\rangle+|-\alpha\rangle)$, and coherent state $|\alpha\rangle$. We observe that the revival time is larger for the cat state than the revival time for the compass state and the revival time is larger for the coherent state than the revival time for cat states. The reduction in revival times is of increasing granular nature of photon distribution from the coherent state to compass state.

\section{APPENDIX B}

In this appendix we follow the argument of Davidovich et al. [3] to show that the detection efficiency is not a serious issue. After passing the first atom $A$ through the cavity, the field state is projected

$$
\left|C_{A}\right\rangle=N\left(e^{i \eta_{1}+\pi / 4}\left|\alpha e^{i \pi / 4}\right\rangle+e^{i \theta_{1}}\left|\alpha e^{-i \pi / 4}\right\rangle\right),
$$

where the velocity of atom $A$ is selected such that the phase change in the cavity field $\phi=\pi / 4$. If one atom similar to atom $A$ passes through the cavity undetected, the combined state will be

$$
\begin{aligned}
\left|\psi^{\prime}\right\rangle= & N e^{i\left(\eta_{1}+\pi / 4\right)}\left(e^{i \eta_{1}+\pi / 4}\left|\alpha^{\prime} e^{i \pi / 2}\right\rangle+e^{i \theta_{1}}\left|\alpha^{\prime}\right\rangle\right)|e\rangle \\
& +N e^{i \theta_{1}}\left(e^{i \eta_{1}+\pi / 4}\left|\alpha^{\prime}\right\rangle+e^{i \theta_{1}}\left|\alpha^{\prime} e^{-i \pi / 2}\right\rangle\right)|g\rangle,
\end{aligned}
$$

where $\alpha^{\prime}=\alpha e^{-i \delta t_{1}}$. We trace out the atomic state as the atom passes undetected, the cavity field will be in the state

$$
\left|C_{A}^{\prime}\right\rangle=N^{\prime}\left[e^{i \eta_{1}+\pi / 4}\left(\left|\alpha^{\prime}\right\rangle+\left|i \alpha^{\prime}\right\rangle\right)+e^{i \theta_{1}}\left(\left|\alpha^{\prime}\right\rangle+\left|-i \alpha^{\prime}\right\rangle\right)\right] .
$$

Now the second atom $B$ enters the cavity and is detected after passing the cavity in earlier defined states. The velocity of the second atom is chosen such that it changes phase of the cavity field by $\pi / 2$. The detection of a second atom will project the cavity field in the state

$$
\begin{aligned}
\left|C_{B}\right\rangle= & \frac{N^{\prime}}{2}\left[e^{i \eta_{1}+\eta_{2}+3 \pi / 4}\left(\left|\alpha^{\prime \prime} e^{i \pi / 2}\right\rangle+\left|i \alpha^{\prime \prime} e^{i \pi / 2}\right\rangle\right)+e^{i\left(\theta_{1}+\eta_{2}+\pi / 2\right)}\right. \\
& \times\left(\left|\alpha^{\prime \prime} e^{i \pi / 2}\right\rangle+\left|-i \alpha^{\prime \prime} e^{i \pi / 2}\right\rangle\right)+e^{i \eta_{1}+\theta_{2}+\pi / 4}\left(\left|\alpha^{\prime \prime} e^{-i \pi / 2}\right\rangle\right. \\
& \left.\left.+\left|i \alpha^{\prime \prime} e^{-i \pi / 2}\right\rangle\right)+e^{i\left(\theta_{1}+\theta_{2}\right)}\left(\left|\alpha^{\prime \prime} e^{-i \pi / 2}\right\rangle+\left|-i \alpha^{\prime \prime} e^{-i \pi / 2}\right\rangle\right)\right],
\end{aligned}
$$

where $\alpha^{\prime \prime}=\alpha^{\prime} e^{-i \delta t_{2}}$. For earlier defined conditions on phases in the method for preparing the compass state (4), $\theta_{1}=\eta_{1}$ $+\pi / 4$ and $\theta_{2}=\eta_{2}+\pi / 2$, the state (B4) becomes the same as state (22). In a similar way one can see that the prepared state will be a compass state if one atom similar to atom $B$ passes undetected between the atoms $A$ and $B$.
[1] W. P. Schleich, in Quantum Optics in Phase Space (Wiley, New York 2001).

[2] V. Buzek and P. L. Knight, in Progress in Optics, edited by E. Wolf (North-Holland, Amsterdam, 1995), Vol. XXXIV.

[3] L. Davidovich, M. Brune, J. M. Raimond, and S. Haroche, Phys. Rev. A 53, 1295 (1996).

[4] A. Auffeves, P. Maioli, T. Meunier, S. Gleyzes, G. Nogues, M. Brune, J. M. Raimond, and S. Haroche, Phys. Rev. Lett. 91, 230405 (2003).

[5] M. Brune, E. Hagley, J. Dreyer, X. Matre, A. Maali, C. Wunderlich, J. M. Raimond, and S. Haroche, Phys. Rev. Lett. 77, 4887 (1996).

[6] J. M. Raimond, M. Brune, and S. Haroche, Phys. Rev. Lett. 79, 1964 (1997).

[7] C. Monroe, D. M. Meekhof, B. E. King, and D. J. Wineland, Science 272, 1131 (1996).

[8] M. W. Noel and C. R. Stroud, Jr., Phys. Rev. Lett. 77, 1913 (1996).
[9] K. Tara, G. S. Agarwal, and S. Chaturvedi, Phys. Rev. A 47, 5024 (1993).

[10] C. Ottaviani, D. Vitali, M. Artoni, F. Cataliotti, and P. Tombesi, Phys. Rev. Lett. 90, 197902 (2003).

[11] S. J. van Enk, Phys. Rev. Lett. 91, 017902 (2003).

[12] A. Imamoglu, H. Schmidt, G. Woods, and M. Deutsch, Phys. Rev. Lett. 79, 1467 (1997).

[13] G. S. Agarwal and J. Banerji, Phys. Rev. A 64, 023815 (2001).

[14] J. Banerji and G. S. Agarwal, Phys. Rev. A 59, 4777 (1999); G. S. Agarwal and J. Banerji, ibid. 57, 3880 (1998).

[15] J. A. Yeazell and C. R. Stroud, Jr., Phys. Rev. A 43, 5153 (1991).

[16] G. S. Agarwal, R. R. Puri, and R. P. Singh, Phys. Rev. A 56, 2249 (1997).

[17] C. C. Gerry, Phys. Rev. A 53, 3818 (1996).

[18] W. H. Zurek, Nature (London) 412, 712 (2001).

[19] G. S. Agarwal and E. Wolf, Phys. Rev. D 2, 2161 (1970). 